\title{
Etude du développement des nauplii de Diaptomus gracilis, O. Sars, et Diaptomus laciniatus, Lilljeborg
}

par

\author{
Marguerite WUTHRICH \\ Assistante à l'Inspectorat cantonal de la pèche, \\ à Neuchâtel. \\ Avec 15 figures dans le texte
}

Nous trouvons dans le plankton du lac de Neuchâtel deux espèces pélagiques de Diaptomus, soit $D$. gracilis $O$. Sars, et D. laciniatus Lilljeborg. M. A.-A. Quartier, inspecteur cantonal de la pêche, à Neuchâtel, a pensé qu'il serait intéressant de consacrer une étude au développement de leurs nauplii et il a bien voulu me confier cette tâche: je me suis donc attachée à mettre en évidence le caractère de chaque stade des deux espèces en dessinant à la même échelle les formes successives.

Pour établir les différences spécifiques, j'ai procédé à de nombreux élevages en partant de femelles œuvées: une femelle était pêchée à la pipette, portée sur un porte-objet et examinée au microscope, plus pour s'assurer qu'il ne se trouvait point dans la goutte d'eau d'œufs détachés ou de nauplius que pour reconnaître l'espèce: avec un peu d'habitude on arrive facilement à distinguer les mâles des femelles, les femelles $D$. gracilis des $D$. laciniatus. Les premières sont en effet plus fines, plus minces, plus colorées que les secondes qui sont plus opaques et d'apparence laiteuse. Les femelles choisies étaient ensuite placées dans un flacon de $13 \mathrm{~cm}$. de hauteur et de $3,5 \mathrm{~cm}$. de diamètre, contenant simplement

Rev. Suisse de Zool., T. 55, 1948. 
de l'eau de robinet. Les nauplii éclos dans les différents flacons m'ont permis d'établir ainsi les différences spécifiques. Ces différences reconnues, c'est avec le produit des pêches effectuées au large du lac de Neuchâtel que nous avons exécuté notre travail. Ces pêches sont faites à l'aide d'un filet à plankton à fermeture, modèle Fuhrmann, soie no 25 , de la surface à 25 mètres. Les carcasses vides trouvées nous ont aidée à vérifier certains détails, cependant les dessins ont été faits avec des animaux conservés au formol et dont les appendices étaient patiemment disséqués. Au reste on retrouve rarement les carcasses des stades I et II, qui sont délicates et se corrompent facilement.

Le stade I a pu être dessiné à son éclosion: lors d'un essai d'élevage de palées fait à l'Inspectorat cantonal de la pêche, j'ai constaté que les alevins étaient bourrés d'œufs de Copépodes, j'en ai compté plus de 20 alignés comme des billes dans l'intestin des poissons. Or, en examinant soit le contenu de l'intestin, soit les boudins excrémentiels, je me suis aperçue que ces œufs ne subissaient aucun dommage mais au contraire conservaient toute leur vitalité, en un mot, n'étaient pas digérés et j'ai maintes fois assisté à l'éclosion de ceux-ci. Des boudins excrémentiels, après examen minutieux, furént placés dans des flacons d'eau, il s'ensuivit une éclosion importante de nauplii. Il s'est révélé à l'observation que ce n'est pas par choix que les alevins se gavent d'œufs, mais inhabiles à saisir les crustacés, ils n'arrivent souvent qu'à happer leurs œufs tandis que le copépode s'échappe.

\section{Développement des NaUPLiI.}

Ayant placé des Diaptomus en état de copulation dans un bocal, j'ai pu voir que les œufs apparaissent 6 jours après l'accouplement et que les premiers nauplii naissaient le $8 \mathrm{e}$ jour après la copulation; le $9 \mathrm{e}$ jour je trouvais des nauplii à leur deuxième stade, le $12^{\mathrm{e}}$ jour des stades III, le $14^{\mathrm{e}}$ des stades IV, puis le rythme ralentit et ce n'est qu'au $25^{\mathrm{e}}$ jour que le stade $\mathrm{V}$ apparaissait. Les cultures n'ont jamais permis que le stade VI s'accomplisse, mais par le moyen de la statistique des pêches il semble que dans le lac le sixième stade apparaît environ 30 jours après le premier.

Les femelles $D$. gracilis portent de 4 à 6 œufs dont le diamètre varie dans les limites de 128 à $144 \mu$, tandis que $D$. laciniatus porte 6 à 10 œufs d'un diamètre variant de 144 à $176 \mu$. 
A l'éclosion les addendes sont agglutinés mais la mucosité se dissout rapidement et les soies s'étalent.

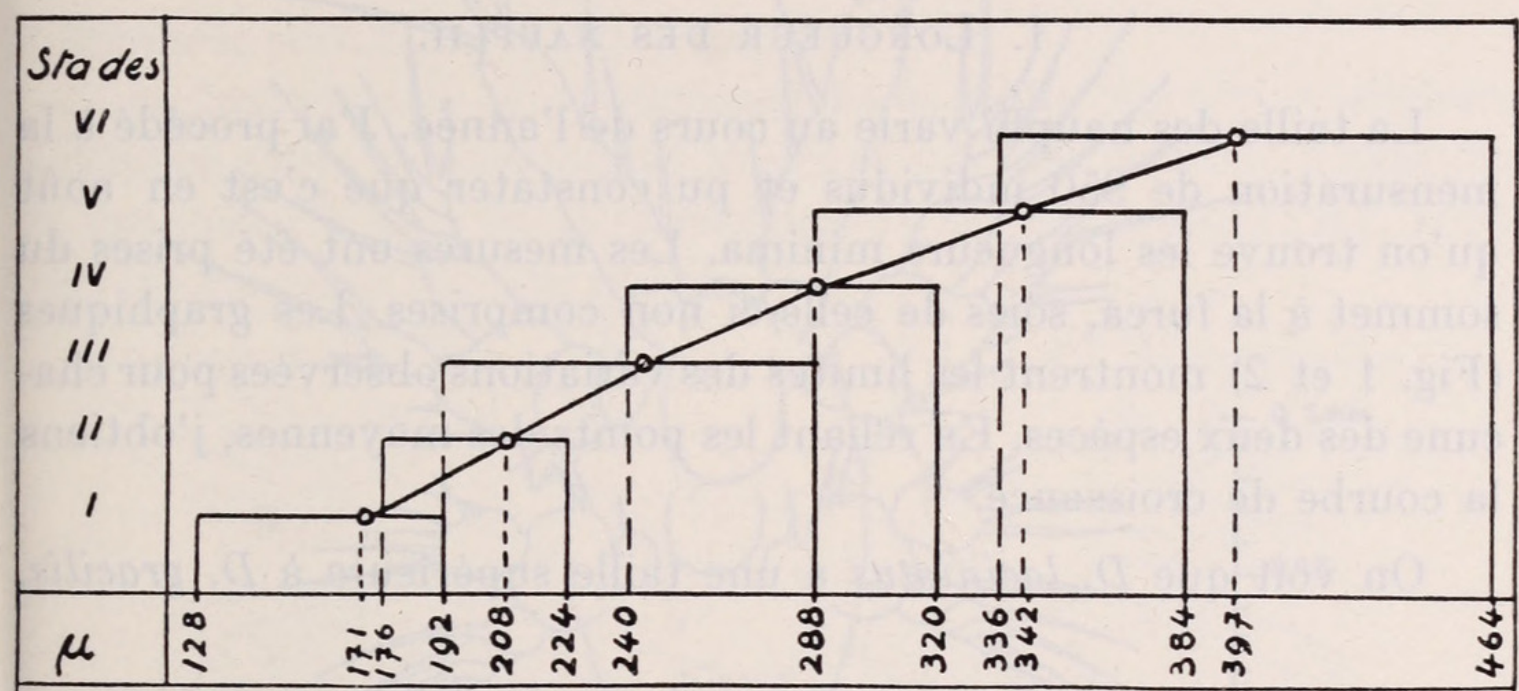

Diaptomus gracills.- Mensuration des Nauplii aux différents stades.

Limites et moyennes des grandeurs. Courbe de croissance.

FIG. 1.

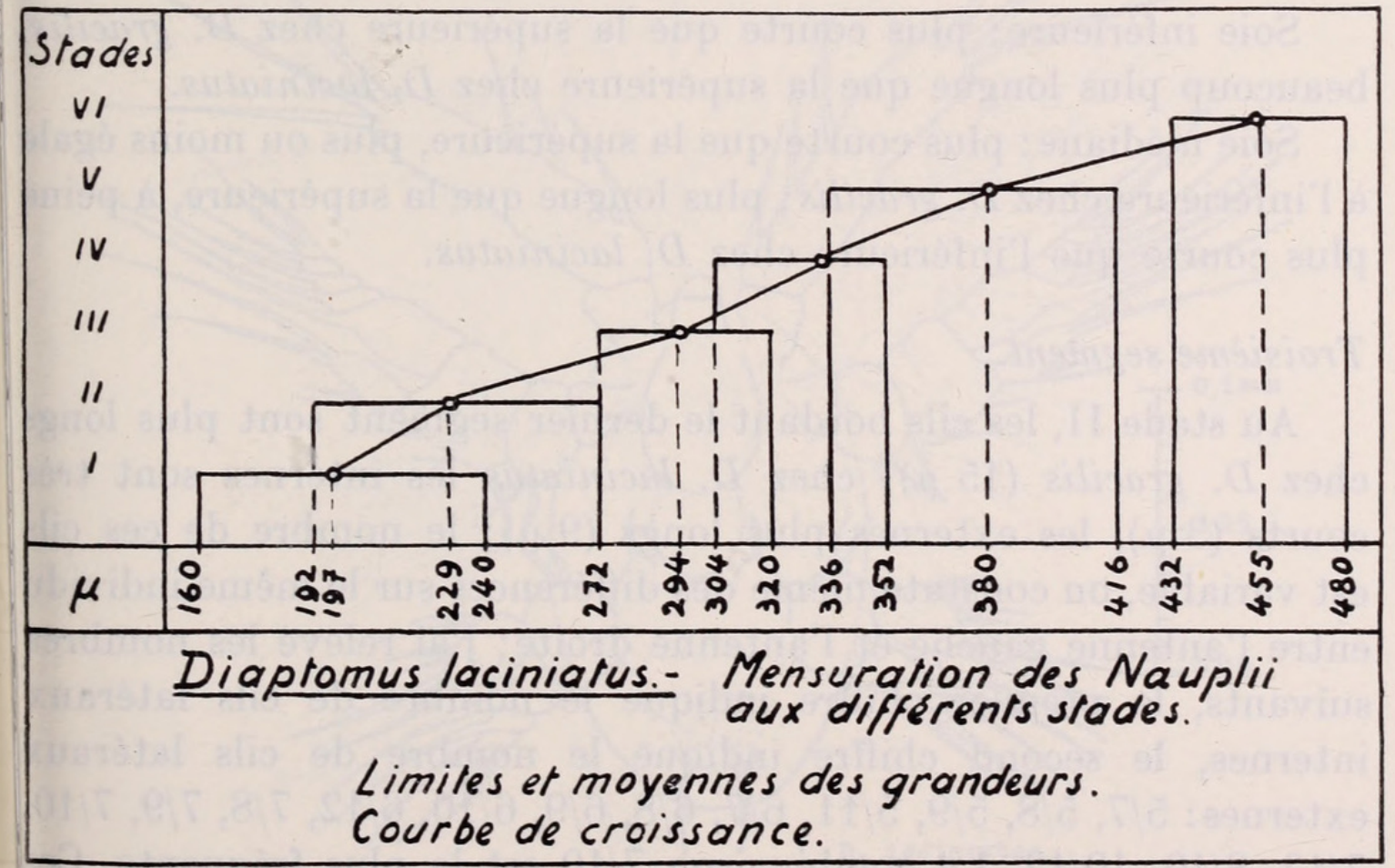

FIG. 2. 
La différence spécifique des nauplii est marquée:

1) par la taille des nauplii,

2) par les soies de la première antenne.

\section{Longueur des Nauplit.}

La taille des nauplii varie au cours de l'année. J'ai procédé à la mensuration de 950 individus et pu constater que c'est en août qu'on trouve les longueurs minima. Les mesures ont été prises du sommet à la furca, soies de celle-ci non comprises. Les graphiques (Fig. 1 et 2) montrent les limites des variations observées pour chacune des deux espèces. En reliant les points des moyennes, j'obtiens la courbe de croissance.

On voit que $D$. laciniatus a une taille supérieure à $D$. gracilis.

\section{SoIES DE LA PREMIÈre ANTENNE.}

C'est en examinant les segments 2 et 3 de la première antenne qu'on verra le plus clairement la différence spécifique, toutefois au stade I, cette différence est très peu marquée.

\section{Deuxième segment.}

Soie inférieure: plus courte que la supérieure chez D. gracilis, beaucoup plus longue que la supérieure chez D. laciniatus.

Soie médiane: plus courte que la supérieure, plus ou moins égale à l'inférieure chez $D$. gracilis; plus longue que la supérieure, à peine plus courte que l'inférieure chez $D$. laciniatus.

\section{Troisième segment.}

$\mathrm{Au}$ stade II, les cils bordant le dernier segment sont plus longs chez $D$. gracilis $(15 \mu)$; chez $D$. laciniatus les internes sont très courts $(3 \mu)$, les externes plus longs $(9 \mu)$; le nombre de ces cils est variable, on constate même des différences sur le même individu entre l'antenne gauche et l'antenne droite; j'ai relevé les nombres suivants, le premier chiffre indique le nombre de cils latéraux internes, le second chiffre indique le nombre de cils latéraux externes: $5 / 7,5 / 8,5 / 9,5 / 11,6 / 7,6 / 8,6 / 9,6 / 10,6 / 12,7 / 8,7 / 9,7 / 10$, $7 / 12,8 / 10,10 / 12$. La combinaison $7 / 10$ est la plus fréquente. Ces cils sont difficiles à compter: ils se plient sur le segment, s'appli- 

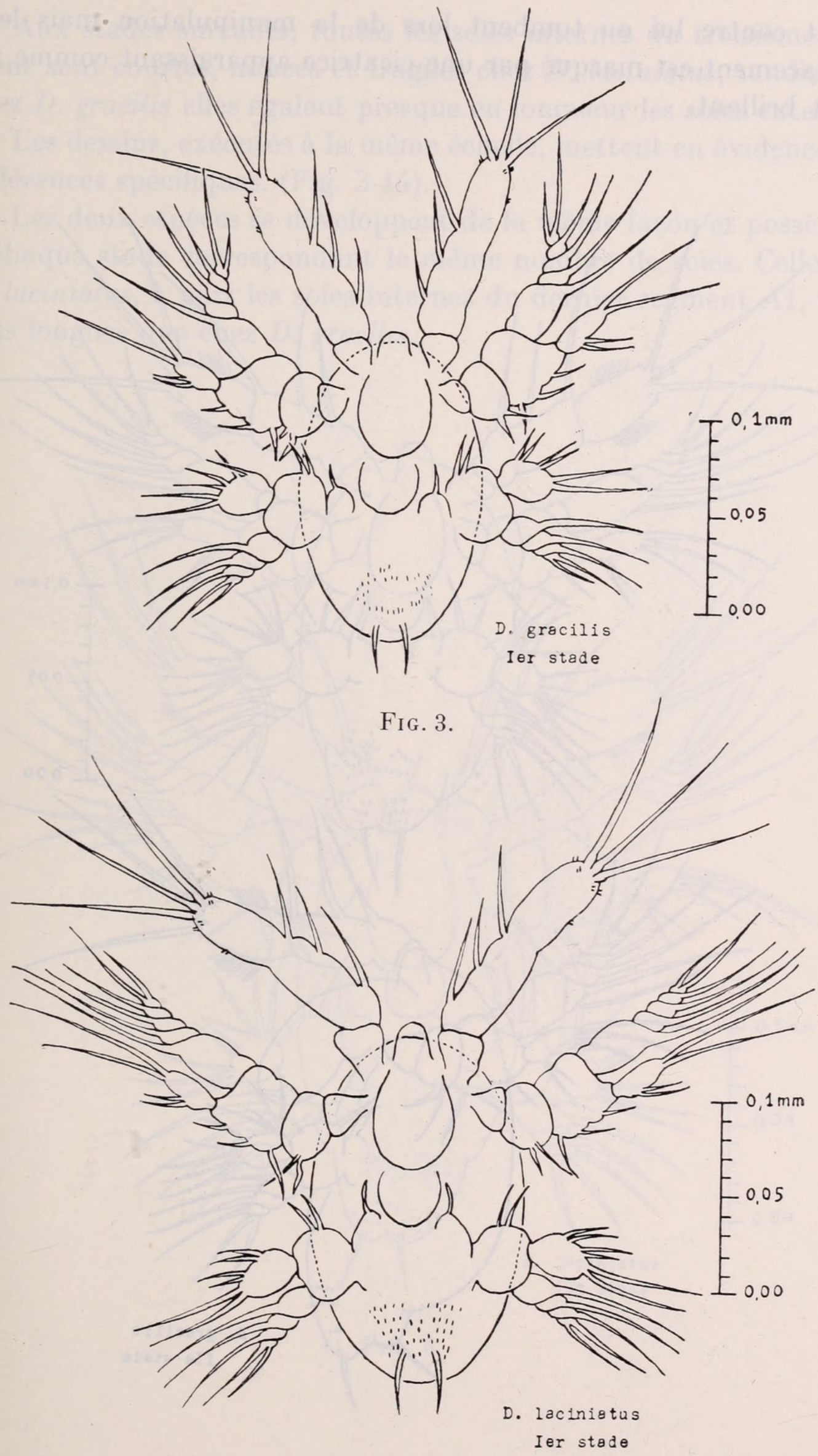

FIG. 4. 
quent contre lui ou tombent lors de la manipulation mais leur emplacement est marqué par une cicatrice apparaissant comme un point brillant.

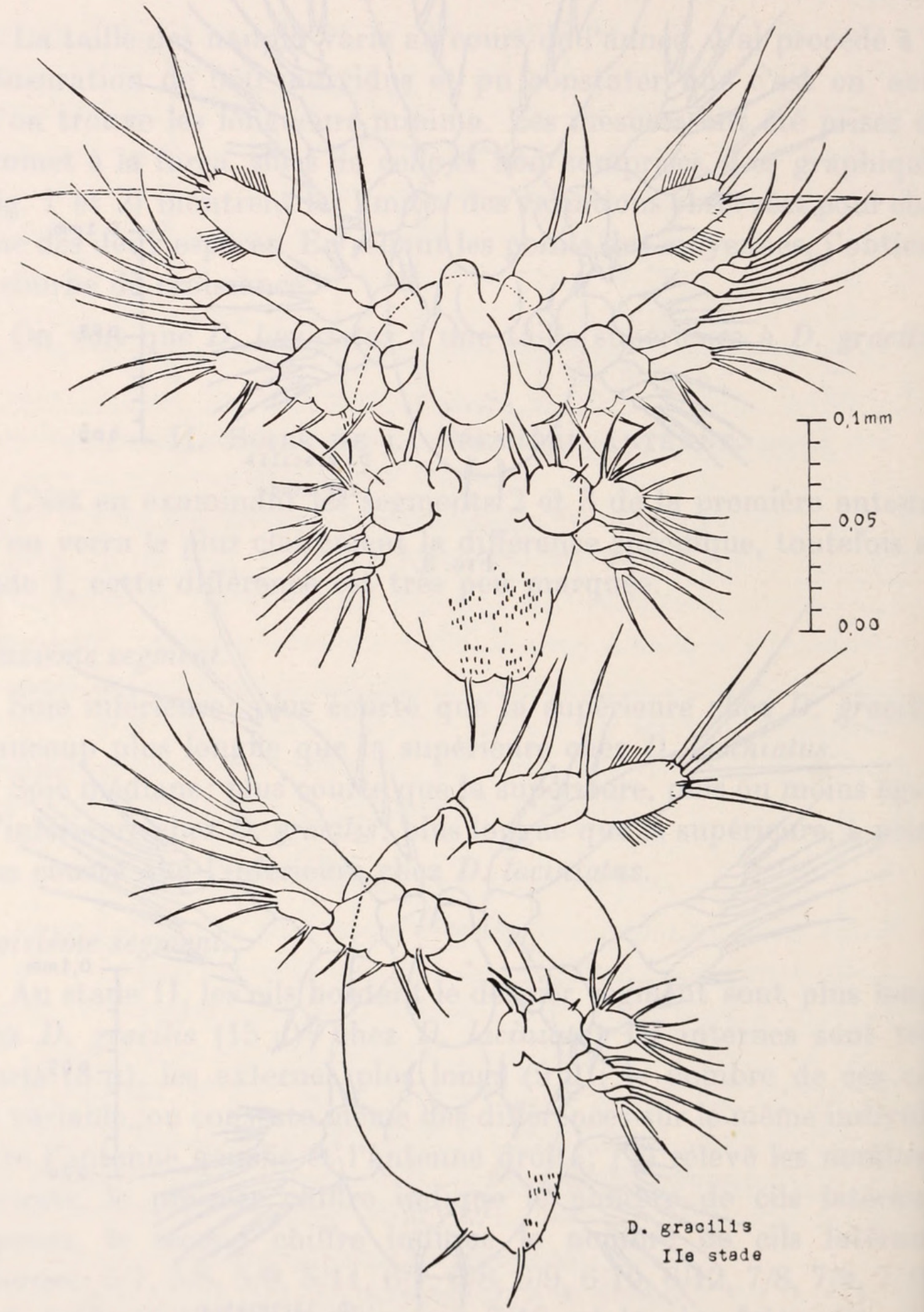

FIG. 5. 
Aux stades suivants, toutes les soies internes du troisième segment sont courtes, minces et fragiles chez D. laciniatus, tandis que chez $D$. gracilis elles égalent presque en longueur les soies externes.

Les dessins, exécutés à la même échelle, mettent en évidence ces différences spécifiques. (Fig. 3-14).

Les deux espèces se développent de la même façon et possèdent à chaque stade correspondant le même nombre de soies. Celles de D. laciniatus, à part les soies internes du dernier segment A1, sont plus longues que chez D. gracilis.

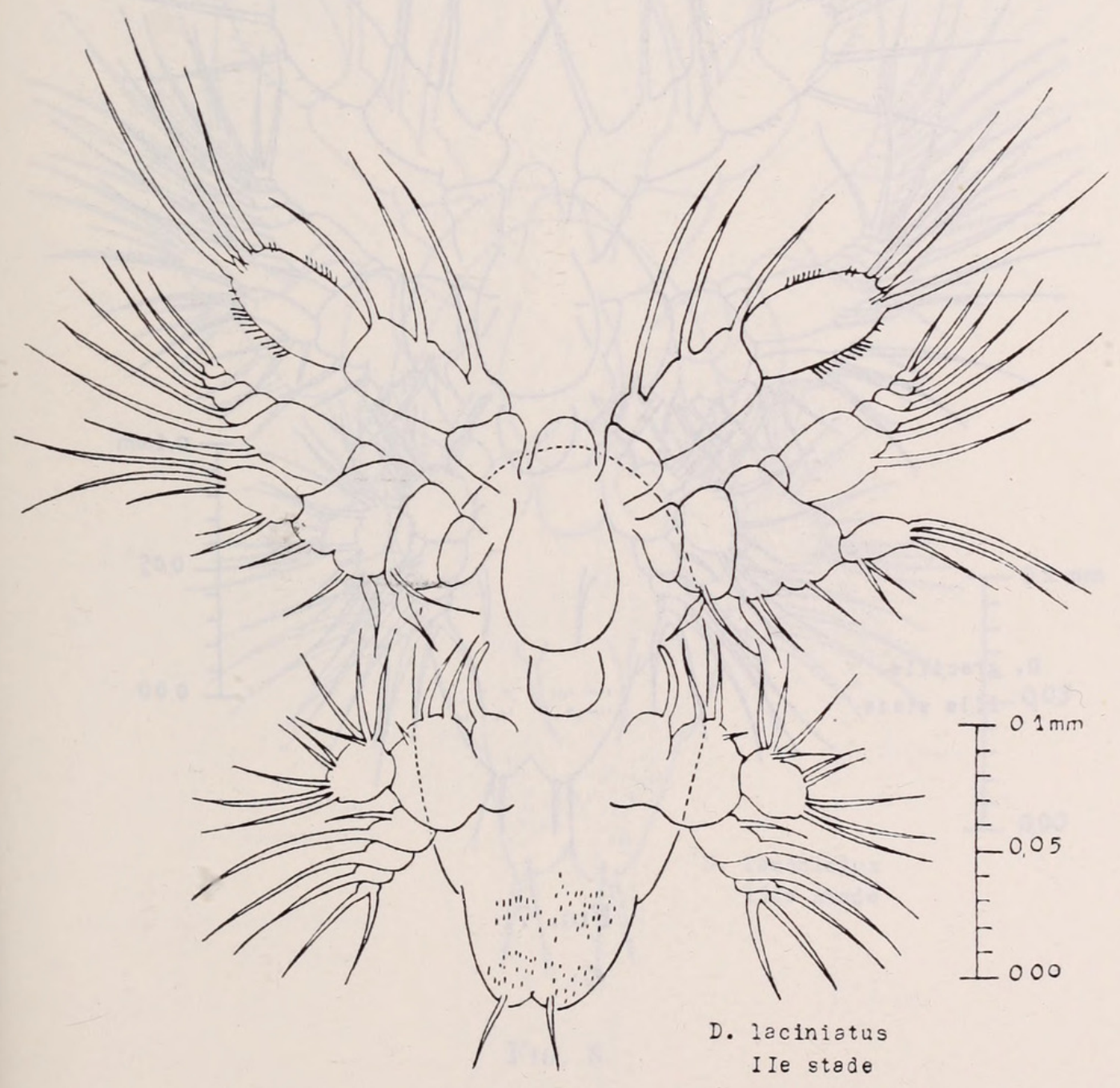

FIG. 6. 


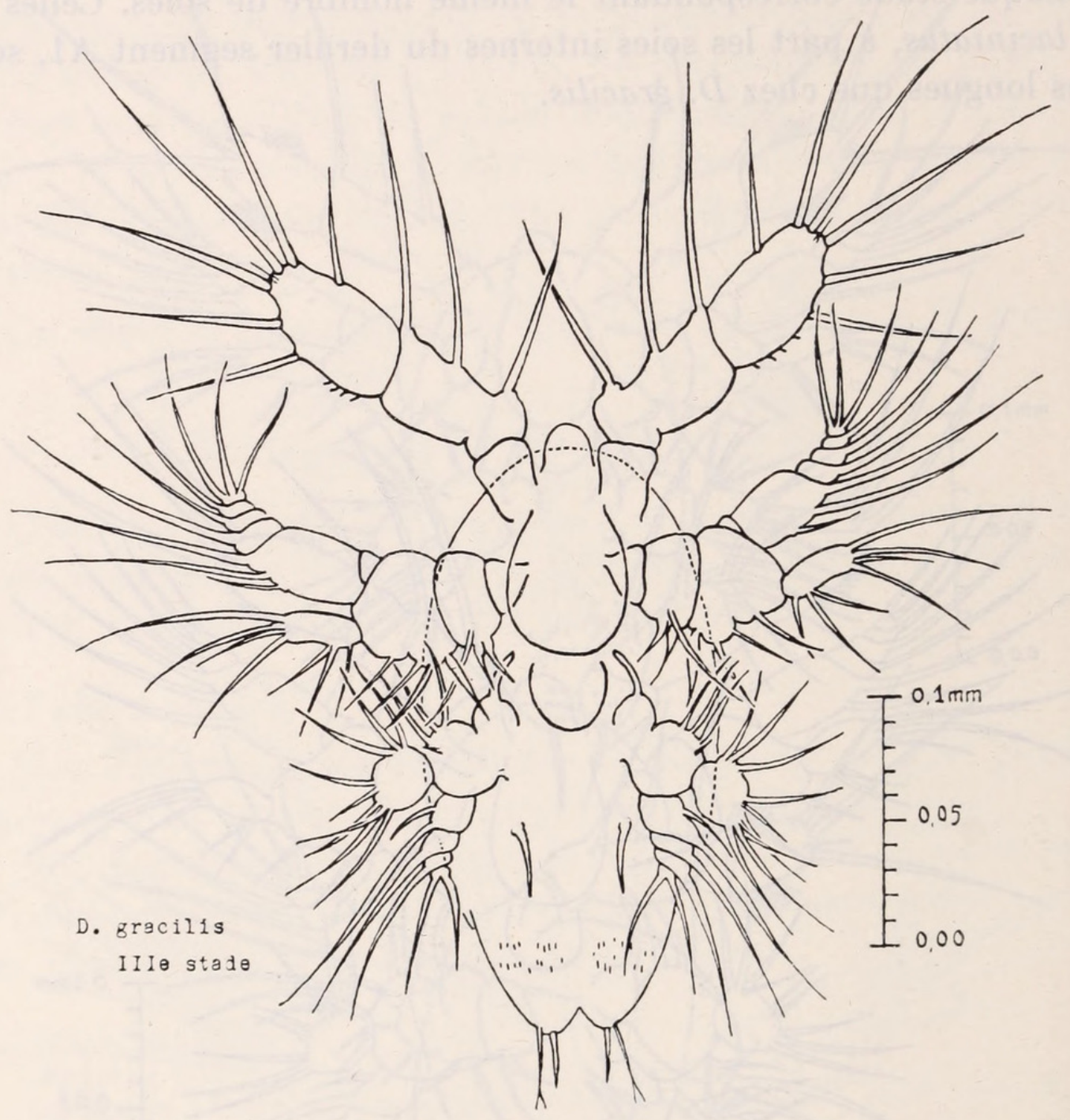

FIG. 7. 


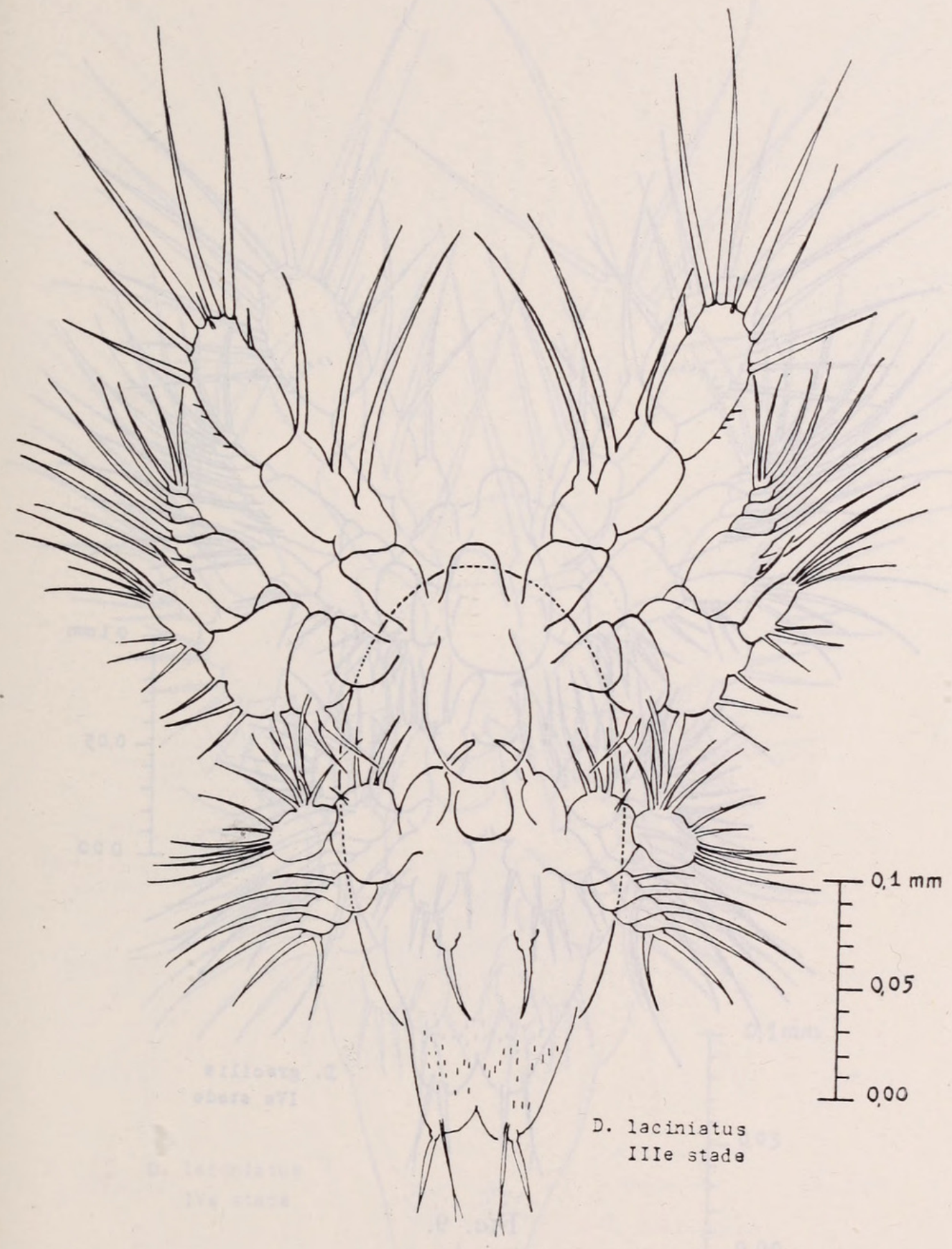

FIG. 8. 


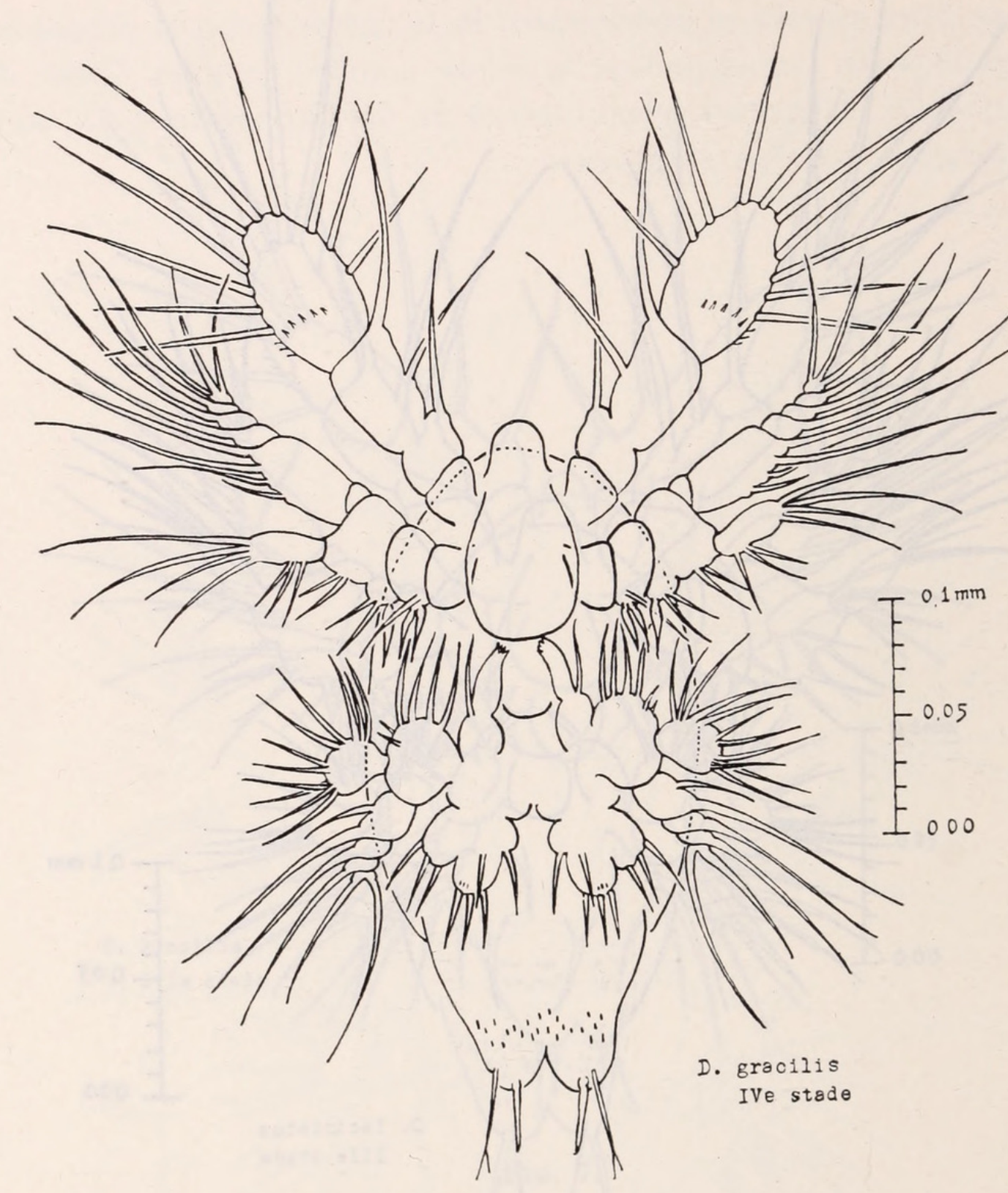

FIG. 9. 


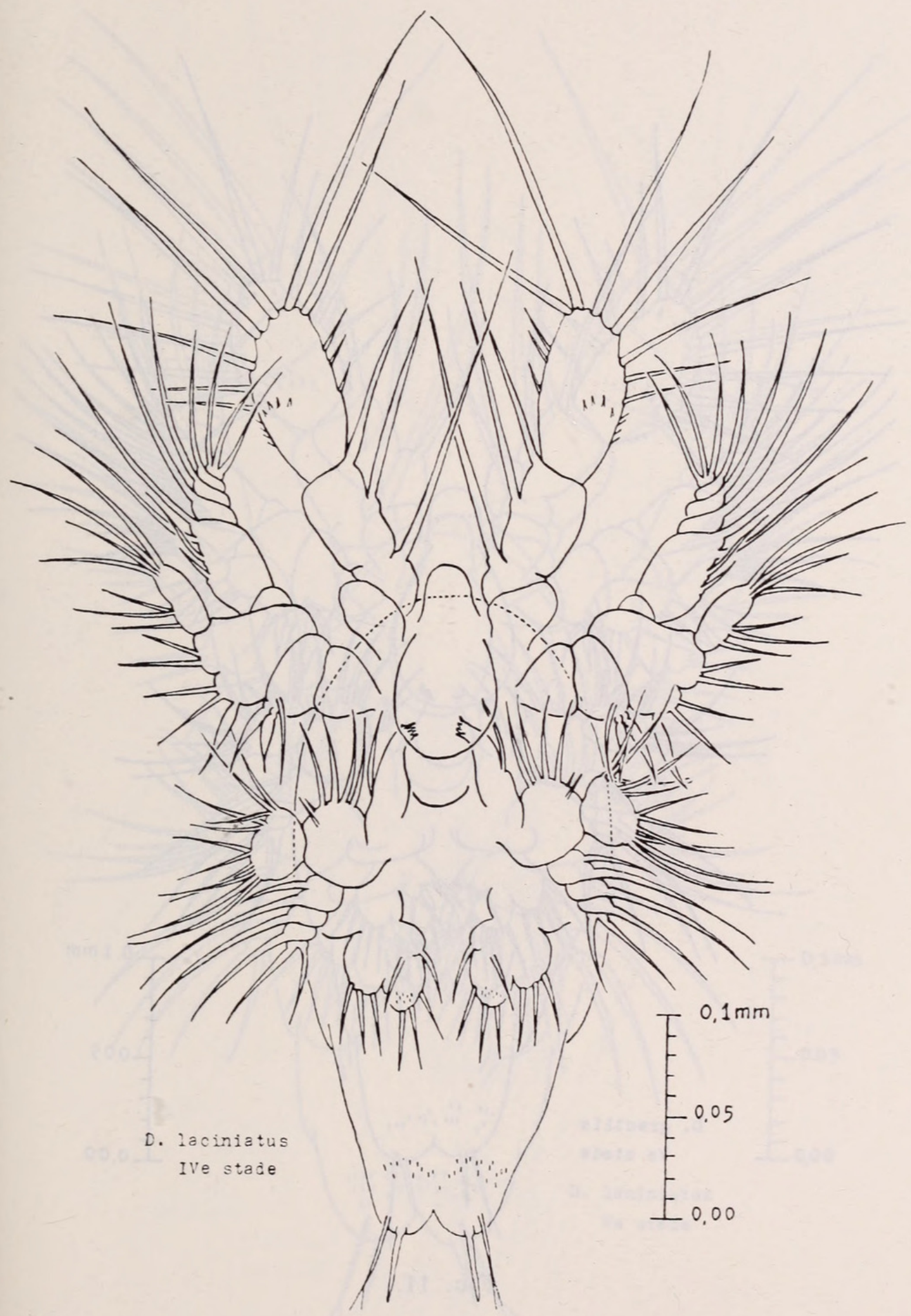

FIG. 10. 


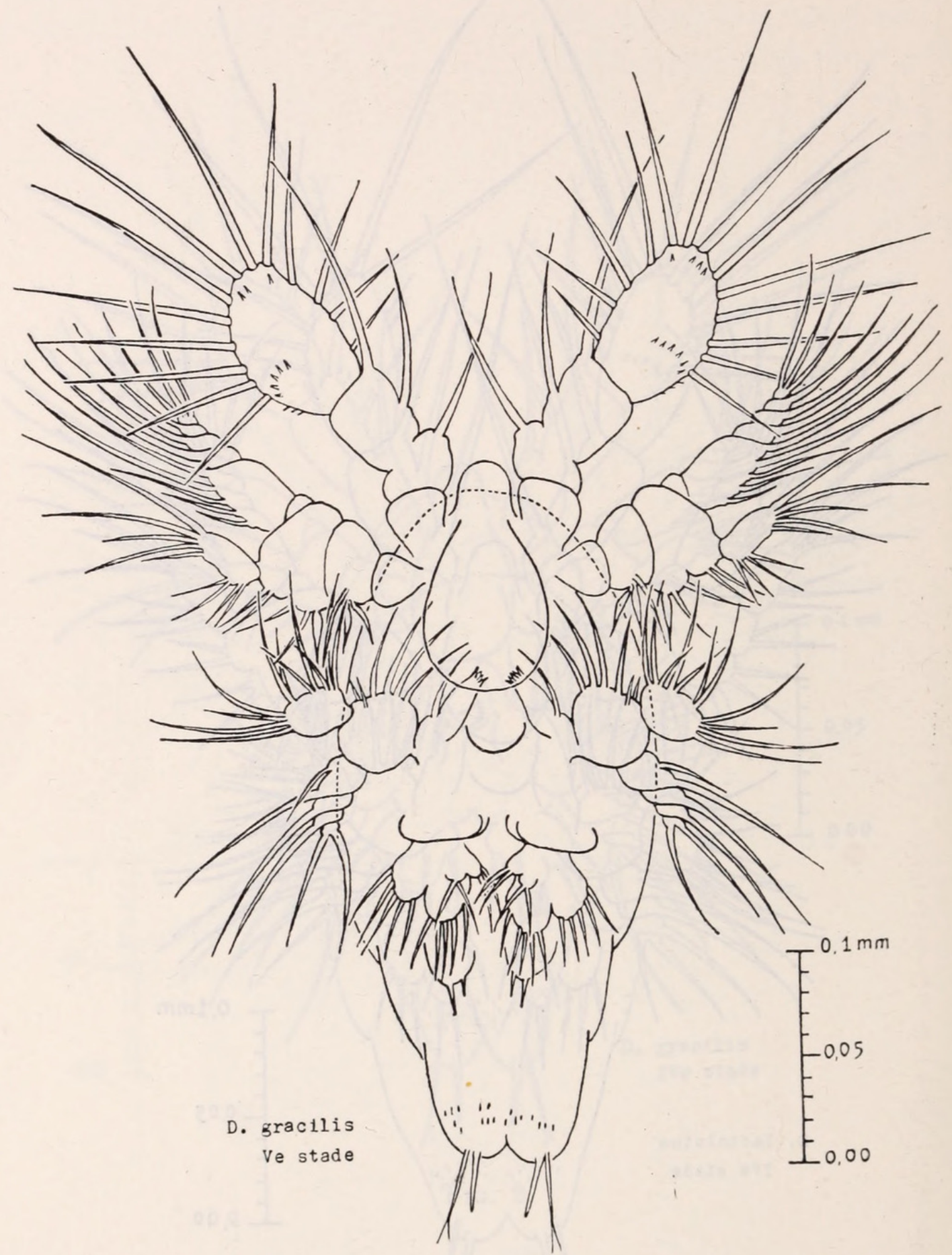

FIG. 11. 


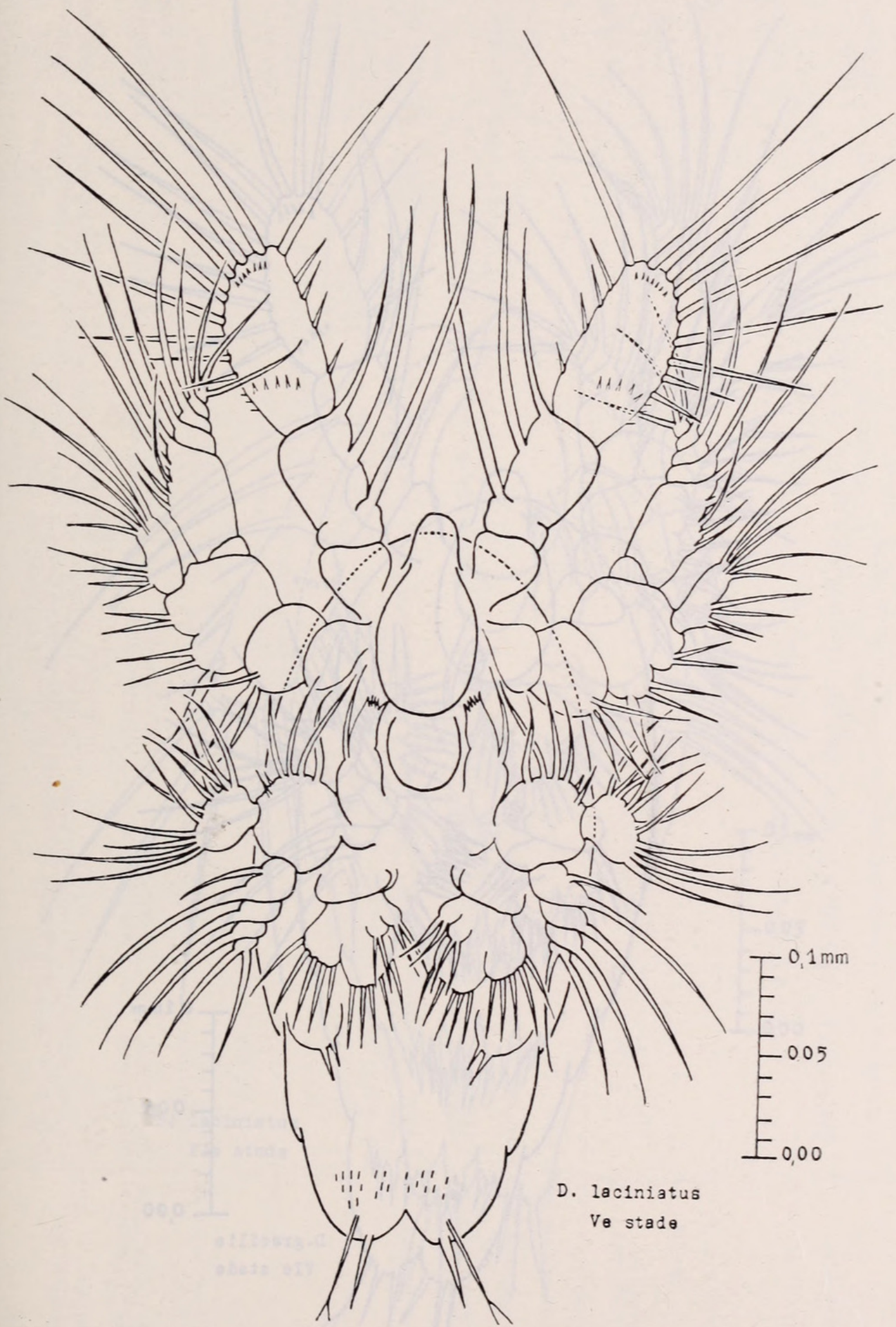

FIG. 12. 


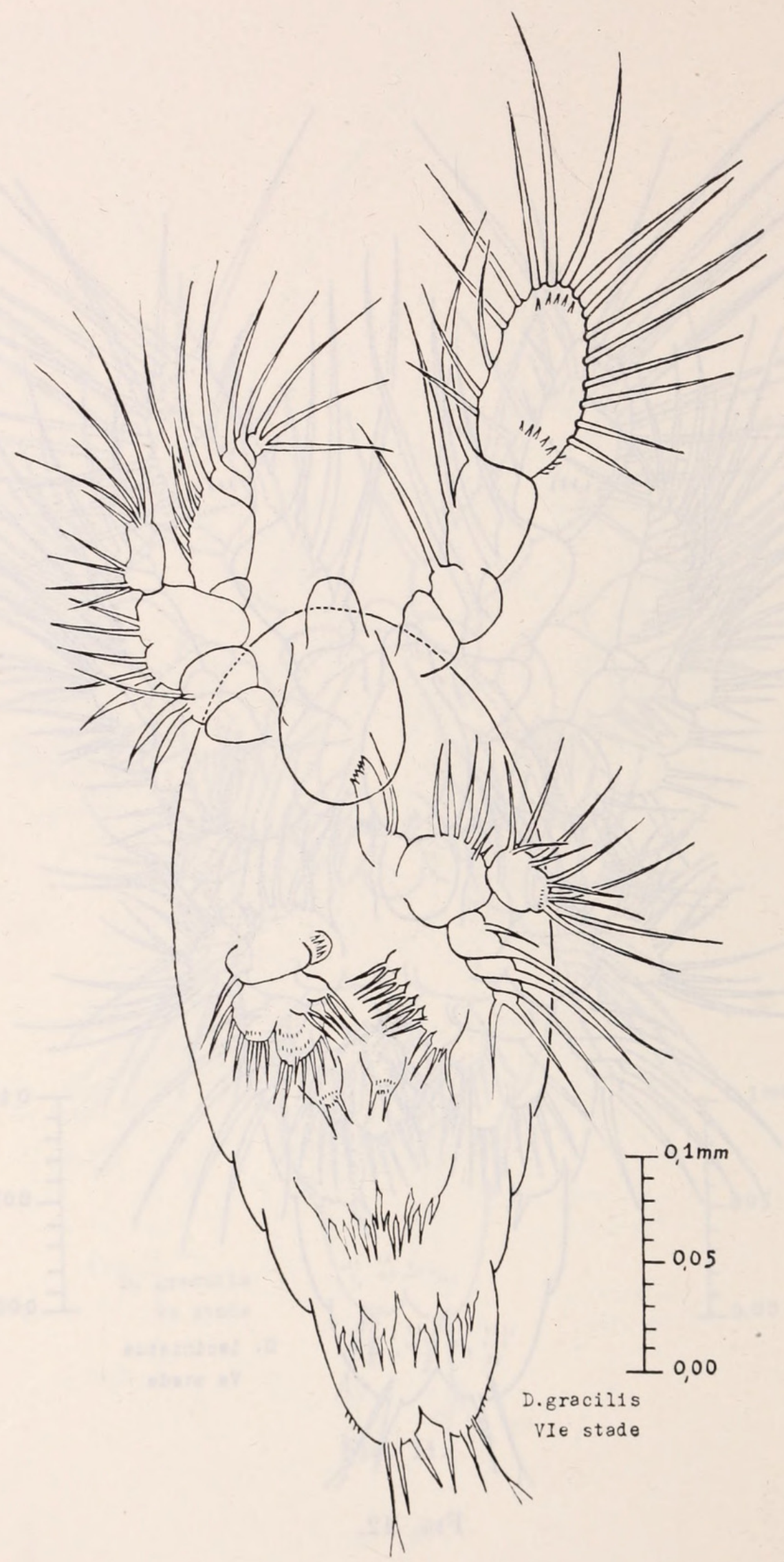

FIG. 13. 


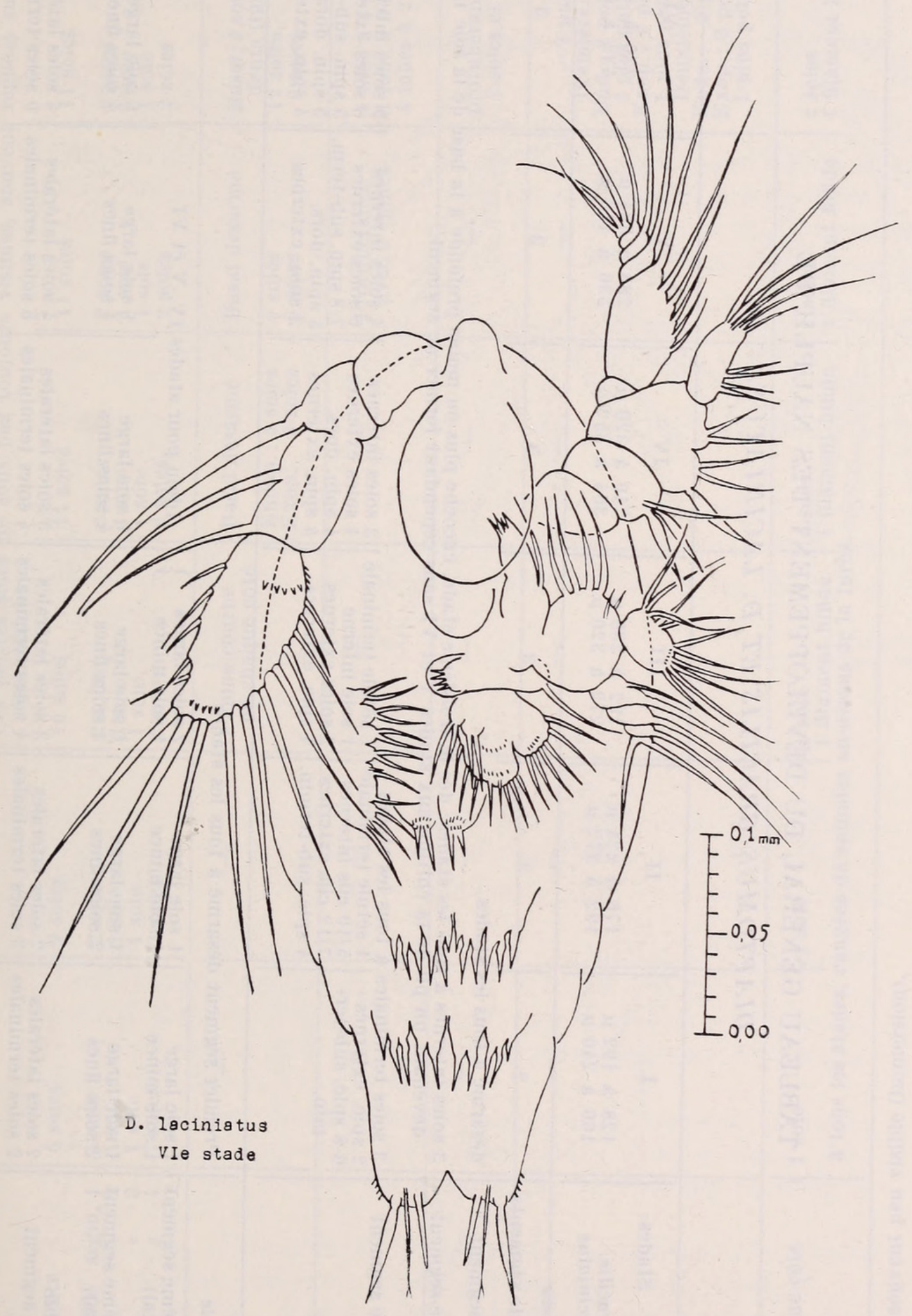

FIG. 14. 


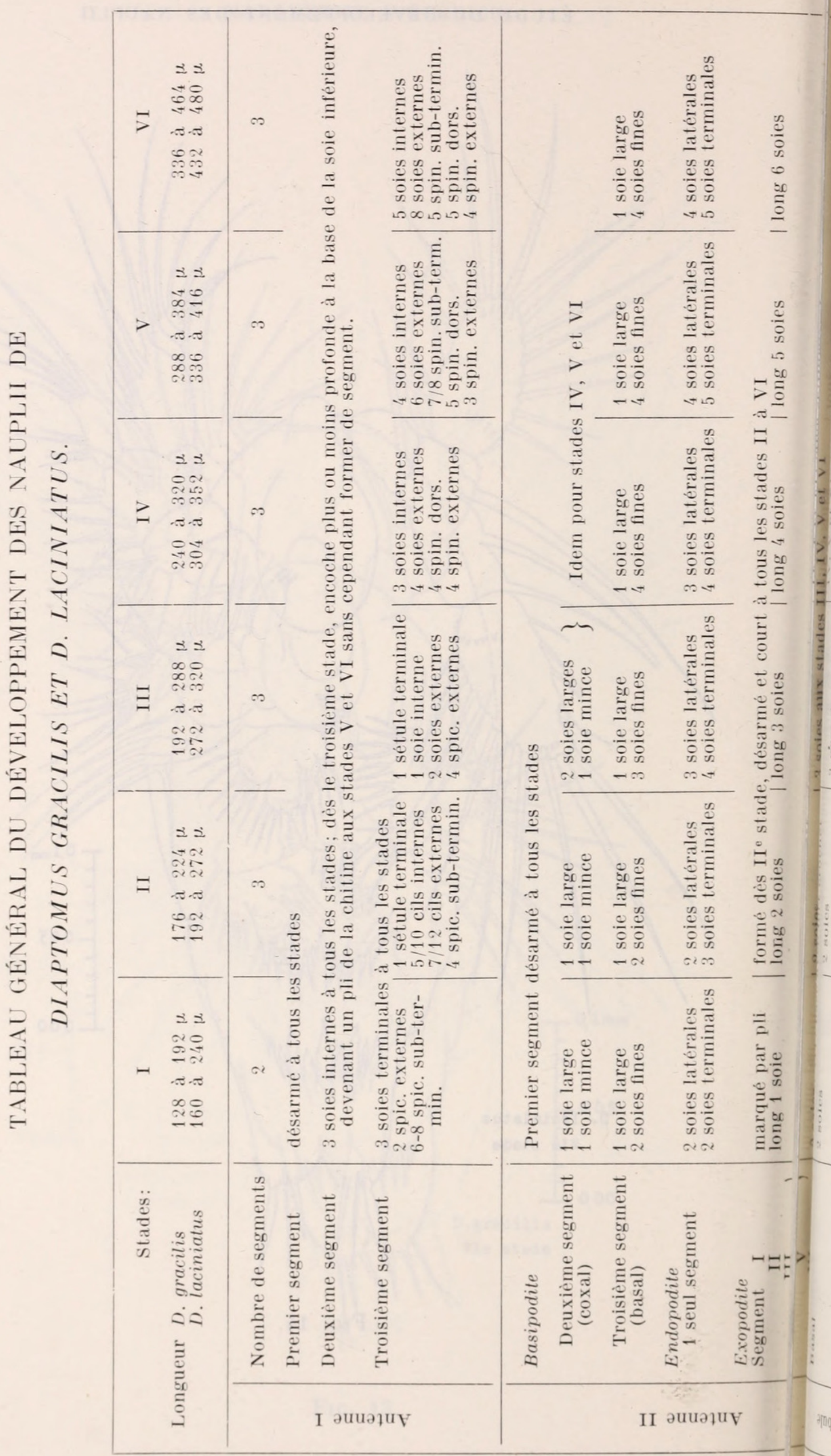



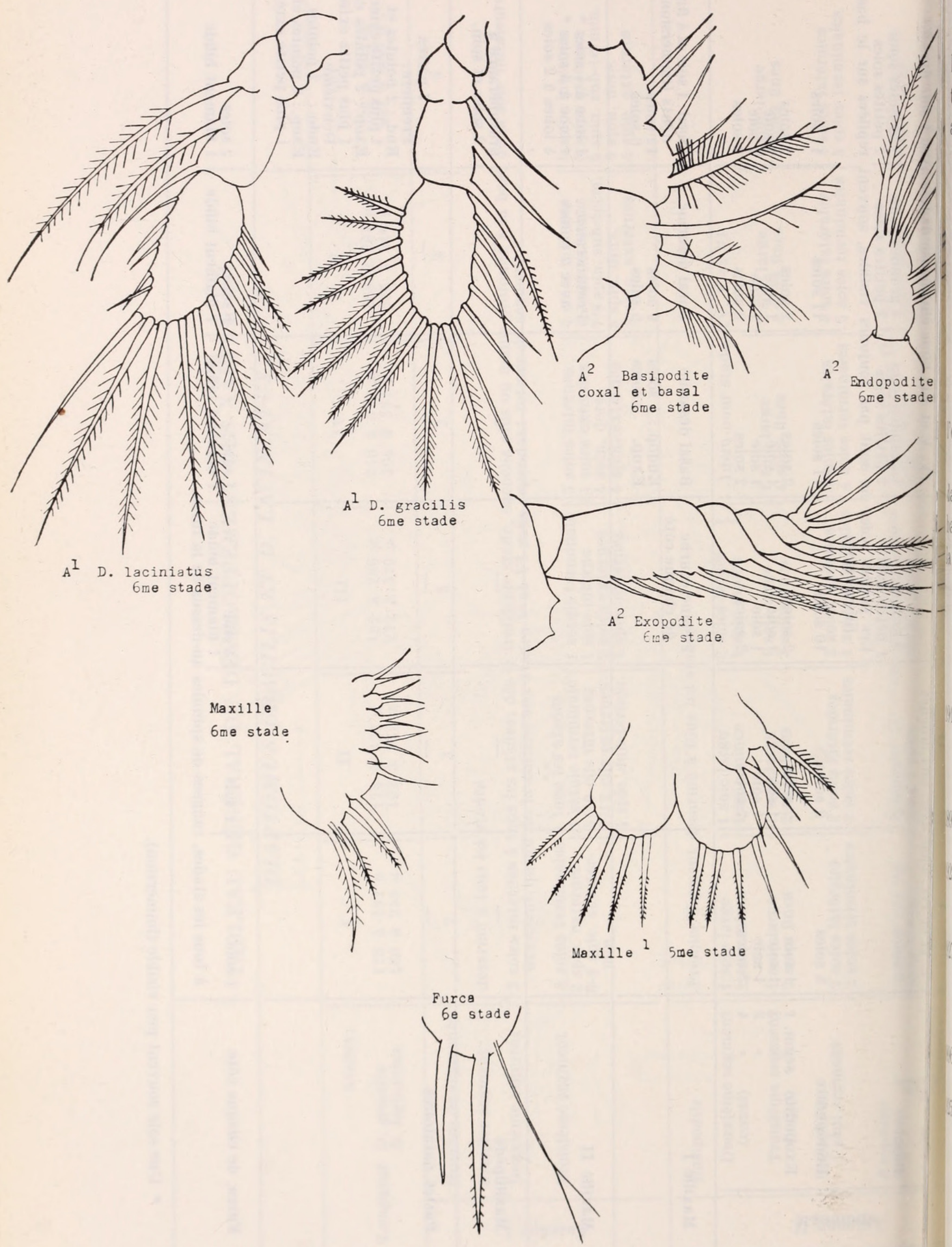

FIG. 15. 
Ces soies sont de différents types: simples, plumeuses ou barbelées. Plutôt que de les décrire toutes, j'ai choisi de faire un dessin très agrandi de certains appendices qui montrera le type des addendes (Fig. 15), soit:

$\mathrm{A}^{1}$ de $D$. lacinialus et de $D$. gracilis, $6^{\text {me }}$ stade;

$\mathrm{A}^{2}$ Basipodite, coxal et basal, Endopodite et Exopodite de $D$. laciniaııs, $6^{\mathrm{me}}$ stade; $D$. gracilis a les soies ornées de la même façon;

Maxille de D. laciniatus, $6^{\mathrm{me}}$ stade; les petites soies du premier et du second lobe sont beaucoup moins facilement visibles chez D. gracilis;

$\mathrm{M}^{1}$ de D. laciniatus, $5^{\mathrm{me}}$ stade; pareil chez $D$. gracilis;

Furca, $6^{\mathrm{me}}$ stade de D. laciniatus; pareille chez D. gracilis.

Le tableau général du développement des nauplii fait ressortir l'ordre d'apparition des appendices.

J'ajouterai encore qu'afin d'avoir des résultats comparables dans les mesures concernant la taille des nauplii, mon travail est basé uniquement sur des pêches bi-mensuelles faites en 1946, de la surface à 25 mètres.

\section{BIBLIOGRAPHIE}

1914. Dietrich, W. Die Metamorphose der freilebenden SüsswasserCopepoden. I. Die Nauplien und das erste Copepodidstadium. Zeitschrift für Wissensch. Zoologie, Bd. 113, p. 252-323; 19 fig.

1898. Giesbrecht, W. und Schmeil, O. Copepoda. I. Gymnoplea. Das Tierreich, herausgeg, v. F. E. Schulze, 6. Lfg.

1928. Gurney, Robert. Dimorphism and state of growth in Copepoda. Internat. Revue der gesammten Hydrologie und Hydrographie, 1928, Bd. XXI, p. 189-207.

927. Rzsoska, Juljian. Einige Beobachtungen über temporale Grössenvariation bei Copepoden und einige andere Fragen ihren Biologie. Internat. Revue der gesammten Hydrologie u. Hydrographie 1927, Bd. XVII, p. 99-114.

896. Schmeil, O. Deutschlands freilebenden Copepoden. III. Teil: Centropagidae. Biblioth. Zoolog. 1896, Bd. VIII, Heft 21.

925. Ziegelmayer, W. Metamorphose und Wachstum der Cyclopiden. Zeitschrift für Wissensch. Zoologie, Bd. 126, p. 493-570; 20 fig., 2 tableaux, 17 tabelles, 15 courbes. 


\section{$2 \mathrm{BHL}$ Biodiversity Heritage Library}

Wuthrich, Marguerite. 1948. "Etude du développement des Nauplii de

Diaptomus gracilis, O. Sars, et Diaptomus laciniatus, Lilljeborg." Revue suisse de zoologie 55, 427-445. https://doi.org/10.5962/bhl.part.117892.

View This Item Online: https://www.biodiversitylibrary.org/item/148889

DOI: https://doi.org/10.5962/bhl.part.117892

Permalink: https://www.biodiversitylibrary.org/partpdf/117892

\section{Holding Institution}

American Museum of Natural History Library

\section{Sponsored by}

BHL-SIL-FEDLINK

\section{Copyright \& Reuse}

Copyright Status: In copyright. Digitized with the permission of the rights holder.

Rights Holder: Muséum d'histoire naturelle - Ville de Genève

This document was created from content at the Biodiversity Heritage Library, the world's largest open access digital library for biodiversity literature and archives. Visit BHL at https://www.biodiversitylibrary.org. 